\title{
Development of expression vectors based on pepino mosaic virus
}

Raquel N Sempere ${ }^{1,2}$, Pedro Gómez ${ }^{1,3}$, Verónica Truniger $^{1}$, Miguel A Aranda ${ }^{\text {* }}$

\begin{abstract}
Background: Plant viruses are useful expression vectors because they can mount systemic infections allowing large amounts of recombinant protein to be produced rapidly in differentiated plant tissues. Pepino mosaic virus (PepMV) (genus Potexvirus, family Flexiviridae), a widespread plant virus, is a promising candidate expression vector for plants because of its high level of accumulation in its hosts and the absence of severe infection symptoms. We report here the construction of a stable and efficient expression vector for plants based on PepMV.

Results: Agroinfectious clones were produced from two different PepMV genotypes (European and Chilean), and these were able to initiate typical PepMV infections. We explored several strategies for vector development including coat protein (CP) replacement, duplication of the CP subgenomic promoter (SGP) and the creation of a fusion protein using the foot-and-mouth disease virus (FMDV) 2A catalytic peptide. We found that CP replacement vectors were unable to move systemically and that vectors with duplicated SGPs (even heterologous SGPs) suffered from significant transgene instability. The fusion protein incorporating the FMDV 2A catalytic peptide gave by far the best results, maintaining stability through serial passages and allowing the accumulation of GFP to 0.2$0.4 \mathrm{~g}$ per $\mathrm{kg}$ of leaf tissue. The possible use of PepMV as a virus-induced gene silencing vector to study gene function was also demonstrated. Protocols for the use of this vector are described.

Conclusions: A stable PepMV vector was generated by expressing the transgene as a CP fusion using the sequence encoding the foot-and-mouth disease virus (FMDV) 2A catalytic peptide to separate them. We have generated a novel tool for the expression of recombinant proteins in plants and for the functional analysis of virus and plant genes. Our experiments have also highlighted virus requirements for replication in single cells as well as intercellular and long-distance movement.
\end{abstract}

\section{Introduction}

Pepino mosaic virus (PepMV) is a widespread plant virus that causes a major disease of tomato crops worldwide [1-11]. PepMV belongs to the genus Potexvirus (family Flexiviridae) and, like other members of this genus, has virions that are non-enveloped flexuous rods $508 \mathrm{~nm}$ in length [12]. The PepMV genome is a singlestranded RNA molecule approximately $6.4 \mathrm{~kb}$ in length, comprising five open reading frames (ORFs) flanked by 5' and 3' untranslated regions (UTRs) with a 5'-cap and a 3' poly-A tail. The ORFs encode a 164-kDa RNAdependent RNA polymerase ( $R \mathrm{dRp})$; three triple gene

\footnotetext{
* Correspondence: m.aranda@cebas.csic.es

'Departamento de Biología del Estrés y Patología Vegetal, Centro de Edafología y Biología Aplicada del Segura (CEBAS)- CSIC, PO Box 164, 30100 Espinardo, Murcia, Spain

Full list of author information is available at the end of the article
}

block (TGB) proteins named TGBp1 (26 kDa), TGBp2 $(14 \mathrm{kDa})$ and TGBp3 $(9 \mathrm{kDa})$; and a $25-\mathrm{kDa}$ coat protein (CP) $[1,13]$. Gene expression is thought to be similar to that in other potexviruses, i.e. the viral replicase is expressed from the genomic RNA (gRNA) whereas the TGB proteins are expressed from subgenomic RNAs (sgRNAs) 1 and 2, and the CP from sgRNA3 [1,13-15]. However, none of the above has been established empirically for PepMV, and little is known about its molecular interactions with host cells.

PepMV is a promising candidate expression vector for plants because of its high level of accumulation in its hosts and the absence of severe infection symptoms for some isolates, factors that have already led to the development of other potexvirus vectors [16,17]. Plant viruses are useful expression vectors because they can mount systemic infections allowing large amounts of recombinant protein to be
C Biomed Central

() 2011 Sempere et al; licensee BioMed Central Ltd. This is an Open Access article distributed under the terms of the Creative Commons Attribution License (http://creativecommons.org/licenses/by/2.0), which permits unrestricted use, distribution, and reproduction in any medium, provided the original work is properly cited. 
produced rapidly in differentiated plant tissues, which makes them particularly suitable for the production of subunit vaccines [18-22]. However, they are also useful for basic research, e.g. as virus-induced gene silencing (VIGS) tools for reverse genetic studies of gene function [23-29] or to investigate the molecular basis of plant-virus interactions $[16,30,31]$.

Many different approaches have been used to convert viruses into expression vectors, including gene replacement, gene insertion, epitope presentation and complementation [16,17,32-36]. Vectors based on tobacco mosaic virus (TMV), potato virus X (PVX), alfalfa mosaic virus (AMV), cucumber mosaic virus (CMV), and cowpea mosaic virus (CPMV) have been engineered as expression vectors and used to produce functional recombinant proteins including vaccines, antigens and enzymes [32,34,37-45]. Early vectors suffered from limitations such as instability and low yields, but this has been addressed by the genetic modification of vector sequences and by delivering virus vectors into plant cells using Agrobacterium tumefaciens [39,40].

Here we describe the development of a PepMV-based vector capable of systemic reporter gene expression in Nicotiana benthamiana plants. We explored different strategies to achieve stable transgene expression, including CP gene substitution, duplication of the CP subgenomic promoter (SGP) and the creation of a translational gene fusion. A stable PepMV vector was generated by expressing the transgene as a $\mathrm{CP}$ fusion using the sequence encoding the foot-and-mouth disease virus (FMDV) 2A catalytic peptide to separate them. We have generated a novel tool for the expression of recombinant proteins in plants, for the analysis of PepMV-plant interactions and for gene function studies. Our experiments have also highlighted virus requirements for replication in single cells as well as intercellular and long-distance movement.

\section{Results}

\section{Agroinfectious PepMV clones}

Isolates PepMV-Sp13 (European tomato genotype) and PepMV-Ps5 (Chilean genotype) [3,13] were used to produce agroinfectious clones [46]. Full-length cDNAs generated by RT-PCR were inserted into vector pBIN61 [47] between the cauliflower mosaic virus (CaMV) $35 \mathrm{~S}$ promoter and the nos terminator, generating plasmids pBPepXL6 (for PepMV-Sp13) and pBPepPs5 (for PepMV-Ps5). Agroinfiltration of $N$. benthamiana leaves with $A$. tumefaciens containing either of the plasmids revealed that both clones were infectious, and very efficient (100\% of plants were infected in each experiment). Agroinoculated plants developed symptoms identical to those induced by the original virus isolates, including mild mosaics, leaf bubbling and stunting. Plants agroinfiltrated with pBPepXL6 showed mild symptoms by 5 days post inoculation (dpi), whereas pBPepPs 5 induced more severe symptoms, although these did not appear until $10 \mathrm{dpi}$. In both cases, infection was readily transmitted to other plants via sap. Dot-blots of total RNA extracted from systemically infected leaves from all the inoculated plants generated a strong hybridization signal when probed with a PepMV replicase sequence, confirming replication and movement of the virus (data not shown). We elected to develop pBPepXL6 as a vector because infection was faster, but accompanied by milder symptoms.

Deletion or replacement of the PepMV coat protein gene As a first step in vector development, we produced PepMV clones in which the CP gene was either mutated or replaced (Figure 1a). In PepXL6agg the first AUG codon was mutated to abolish CP expression, whereas in PepGFP $\triangle \mathrm{CP}$ most of the $\mathrm{CP}$ gene was replaced with

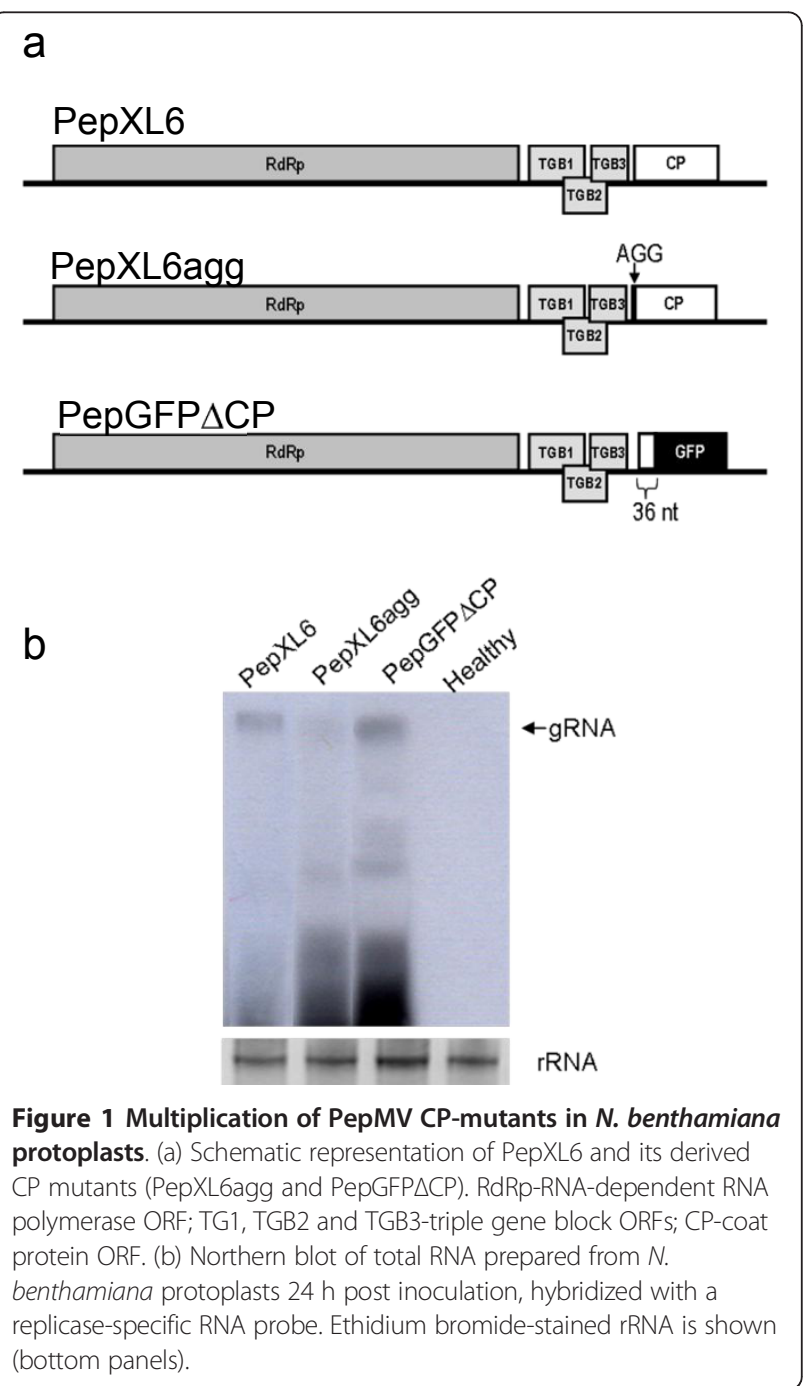


the gene for green fluorescent protein (GFP) to create an in-frame fusion (Figure 1a). We produced two sets of three constructs (PepXL6agg, PepGFP $\triangle \mathrm{CP}$ and wildtype PepXL6), one set retaining the CaMV $35 S$ promoter and nos terminator for expression in planta, and the other using the T7 promoter for run-off in vitro transcription. Northern blots of total RNA extracted from $N$. benthamiana protoplasts transfected with in vitro transcribed viral RNA showed that virus replication efficiency was construct-dependant, with PepGFP $\triangle C P$ accumulating to similar levels as the wild type, but PepXL6agg accumulating in reproducibly lower amounts (Figure 1b). CP expression therefore does not appear necessary for PepMV replication, but the presence of a translatable ORF in the place of the CP ORF appears to promote virus accumulation at the single-cell level.

Next, we agroinfiltrated $N$. benthamiana leaves with PepGFP $\triangle C P$ in the presence of pBp19 (expressing the silencing suppressor P19) to avoid any time-dependant decline in GFP fluorescence [48]. GFP was typically observed in single epidermal and mesophyll cells (Figure 2a, left). Groups of three fluorescent cells were observed occasionally, but the fluorescence did not move to adjacent cells. Importantly, the number of cells expressing GFP in the area agroinfiltrated with pBPepGFP $\triangle C P$ was very low compared to the control construct, $\mathrm{pBGFP}$ (Figure 2a, right). These results confirmed that although the CP is unnecessary for PepMV replication in single cells, it is required for virus movement, as shown for other potexviruses [49-51].

We carried out additional agroinfiltration experiments to determine whether CP provided in trans could complement PepGFP $\triangle \mathrm{CP}$ and restore intercellular movement. This was achieved by co-infiltrating $N$. benthamiana leaves with pBPepGFP $\triangle C P$ and pBINCPep, containing the $\mathrm{CP}$ gene. Confocal laser scanning microscopy revealed that $\sim 90 \%$ of the GFP foci in co-infiltrated leaves consisted of multiple (usually more than nine) cells (Figure $2 b$ ), confirming that PepMV CP movement functions could be provided in trans.

\section{PepMV vectors with a duplicated subgenomic mRNA promoter}

We next designed two full-length PepMV-GFP vectors, each carrying a duplicated CP subgenomic mRNA promoter (SGP). In Pep5.128, the GFP gene was positioned downstream the CP gene, whereas in Pep501 it was positioned upstream (Figure 3a). The duplicated SGP began 76 bp upstream of the CP start codon and ended 36 bp downstream, with the upstream sequence containing the potexvirus-specific octanucleotide 5 'GTTAAGTTT-3' [52,53]. The corresponding cDNAs were inserted into pBIN61 between the CaMV $35 S$ promoter and nos terminator as above, resulting in
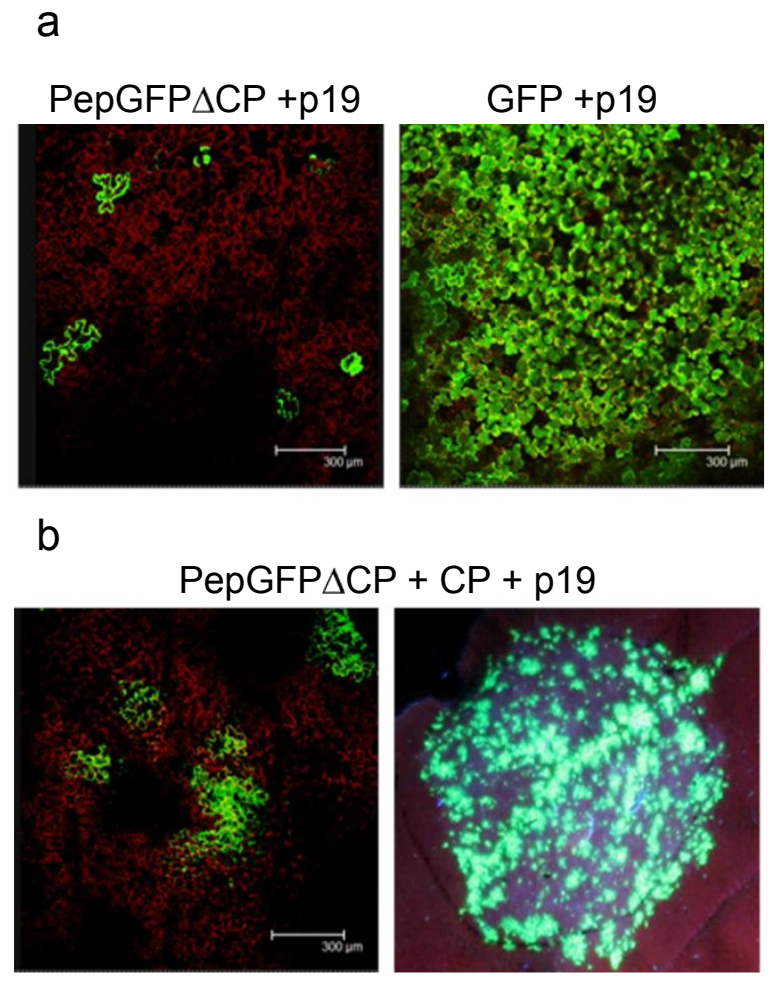

Figure 2 Effect of CP deletion on PepMV multiplication in agroinfiltrated $N$. benthamiana tissues, with and without $C P$ trans complementation. (a) Leaves agroinfiltrated with pBPepGFP $\triangle C P$ (left) and pBGFP (rigth) at 6 days post inoculation (dpi). (b) Leaves co-agroinfiltrated with pBPepGFP $\triangle C P$ and the construct for CP expression, at $6 \mathrm{dpi}$ (left) and $10 \mathrm{dpi}$ (right). The agroinfiltration assays were carried out in the presence of pB19. Photographs at $6 \mathrm{dpi}$ were taken by confocal laser scanning microscopy and the photograph at $10 \mathrm{dpi}$ was taken under illumination with a handheld UV lamp.

constructs pBPep501 and pBPep5.128. N. benthamiana leaves were agroinfiltrated with these plasmids in the presence of pBp19 and GFP expression was monitored from 3-15 dpi. In each case, fluorescence was detected in the agroinfiltrated leaves and systemically by $5 \mathrm{dpi}$, confirming that the mutants were infectious and capable of intercellular movement (Figure 3b). However, virus spreading could only be monitored up to $10 \mathrm{dpi}$ because GFP fluorescence fell below the detection threshold at this time. Northern blots of total RNA extracted from infected plants (agroinfiltrated and systemically-infected leaves) at different time-points revealed a signal corresponding to the predicted size of GFP-CP sgRNA by 5 dpi (Figure 3b, lane 2, marked with star) but this band was no longer visible by $7 \mathrm{dpi}$, another band matching the expected size of the wild type CP sgRNA was observed (Figure 3c, lane 3). This suggested that the GFP transgene had been excised from the vector genome by recombination during the course of the infection. 


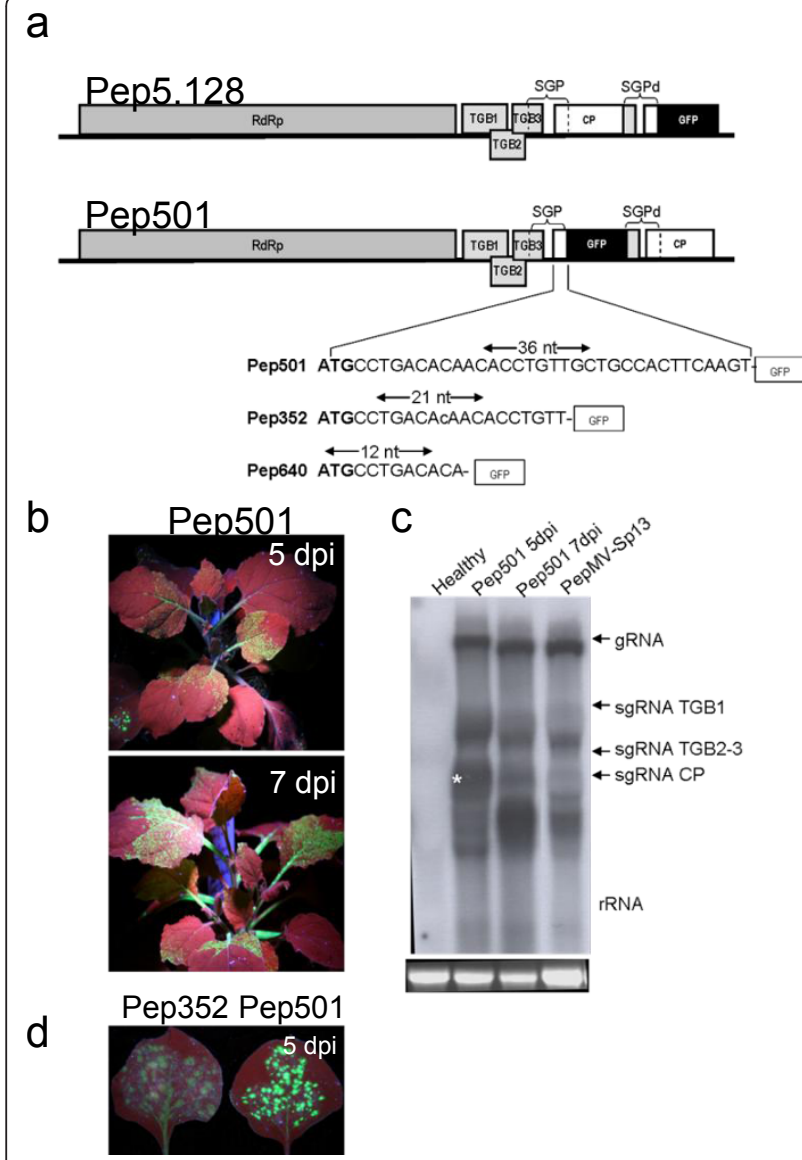

Figure 3 PepMV vectors containing a duplicated subgenomic mRNA promoter. (a) In Pep5.128, the GFP gene was positioned downstream of the CP gene, whereas it was positioned upstream of the CP gene in Pep501. The duplicated SGP spanned positions -76 to +36 relative to the CP start codon. Pep352 and Pep640 include successive deletions at the $3^{\prime}$ end of the putative CP SGP. SGP-CP subgenomic mRNA promoter; SGPd-duplicated CP subgenomic mRNA promoter. (b) Green fluorescence visible in systemicallyinfected $N$. benthamiana leaves from plants agroinfiltrated with pBPep501 at 5 and 7 dpi. (c) Northern blot of leaf tissue hybridized with a CP-specific RNA probe to determine Pep501 stability. Total RNA was prepared from systemically-infected leaves at 5 and $7 \mathrm{dpi}$. The band corresponding to the GFP-CP sgRNA is marked with star. Ethidium bromide-stained rRNA is shown (bottom panels). (d) $\mathrm{N}$. benthamiana leaves infiltrated with pBPep352 or pBPep501 vectors, showing fluorescence emitted under UV illumination with a handheld UV lamp. Photographs were taken at 5 or $7 \mathrm{dpi}$.

In an effort to improve stability, we attempted to reduce the size of the $\mathrm{CP}$ SGP by eliminating sequences from the 3' end (Pep352 contained 21 bp of the CP gene and Pep640 contained $12 \mathrm{bp}$, as shown in Figure $3 a)$. The corresponding cDNAs were inserted into pBIN61 as described above and introduced into $N$. benthamiana leaves by agroinfiltration in the presence of pBp19. No fluorescence was observed in plants inoculated with Pep640 (data not shown), but fluorescence was detected in plants inoculated with Pep352 by 4 dpi both in agroinfiltrated and systemically-infected leaves. However, fluorescence was $55 \%$ less intense than in plants inoculated with Pep501, and began to decline by $7 \mathrm{dpi}$, suggesting even greater instability (Figure 3d).

\section{PepMV vectors with a heterologous CP subgenomic mRNA promoter}

To reduce the likelihood of excision by recombination, we constructed a vector based on PepMV-Sp13 that contained the GFP from the heterologous CP SGP of PepMV-Ps5. In this vector, named Pep505, the GFP gene was placed downstream of the $C P$ gene (Figure 4a). Pep505 was capable of replication and systemic infection in $N$. benthamiana plants but was less efficient than Pep501. Systemic GFP fluorescence was observed by $5 \mathrm{dpi}$ but had begun to decline in upper leaves by 7 dpi (Figure 4b). Northern blots of total RNA from infected plants (agroinfiltrated and systemically-infected leaves) at different time-points were hybridized with a probe specific for the CP gene (Figure 4c), showing the presence of a band of the predicted size for the CP-GFP sgRNA at 7 dpi (Figure 4c, lane 1, marked with star). In systemically-infected leaves, however, this signal began

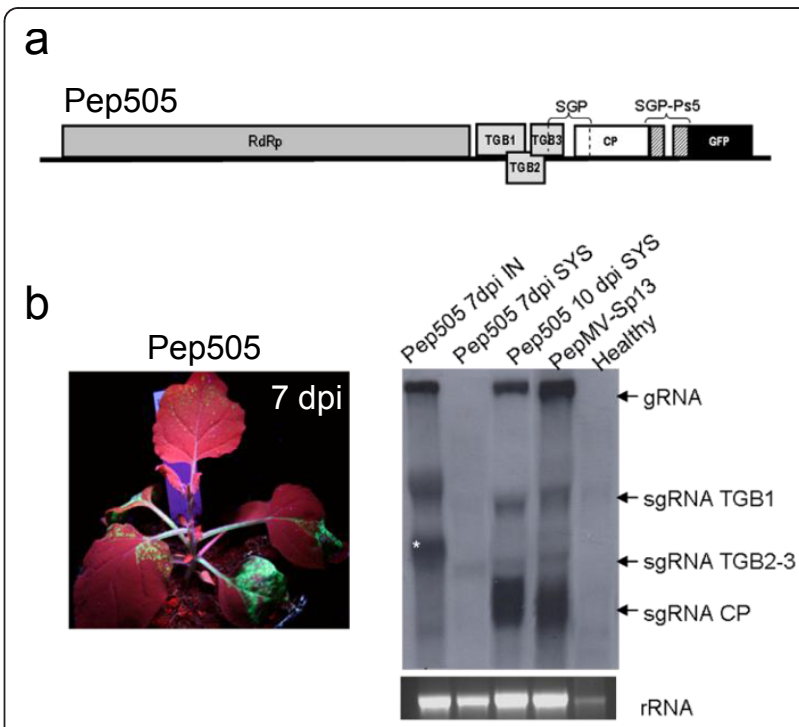

Figure 4 PepMV vectors with a heterologous CP subgenomic mRNA promoter. (a) Schematic representation of Pep505. SGP-Ps5 corresponds to the PepMV Ps5 (Chilean genotype) CP subgenomic mRNA promoter. (b) Fluorescence in systemically-infected leaves of N. benthamiana plants agroinfiltrated with pBPep505. Plants were photographed under illumination with a handheld UV lamp at 7 dpi. (c) Northern blot of total RNA from leaf tissues hybridized with a CP-specific RNA probe to determine Pep505 stability. Total RNA was prepared from agroinfiltrated (IN) and systemically-infected (SYS) leaves at 7 and $10 \mathrm{dpi}$. The band corresponding to the CP-GFP sgRNA is marked with star. Ethidium bromide-stained rRNA is shown (bottom panels). 
to fade between 7 and $10 \mathrm{dpi}$, and was replaced with a signal matching the predicted size of the wild-type $\mathrm{CP}$ gene. The heterologous sequence therefore did not improve vector stability.

\section{PepMV vectors including the FMDV $2 \mathrm{~A}$ catalytic peptide sequence}

In order to avoid sequence duplication, we explored an alternative expression strategy based on a $\mathrm{CP}$ fusion that included the FMDV 2A catalytic peptide sequence [54], resulting in vector pBPepGFP2a (Figure 5a). Following agroinfiltration, green fluorescent spots visible to the naked eye when leaves were illuminated with a handheld UV lamp appeared by 3-4 dpi. Subsequent long-distance movement of the virus to developing leaves led to the appearance of intense green fluorescence in systemically infected leaves 7-10 dpi (Figure 5b). The movement of PepGFP2a was therefore slower than Pep501, where systemic fluorescence was visible 5-6 dpi (Figure 3b). After $12 \mathrm{dpi}$, almost the complete plant expressed fluorescence,

\section{a}

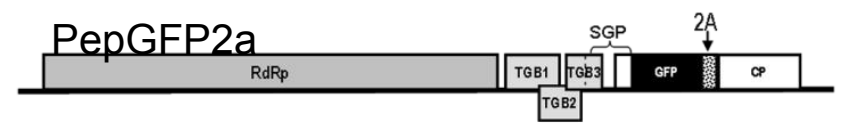

b

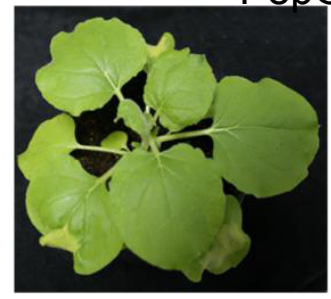

PepGFP2a
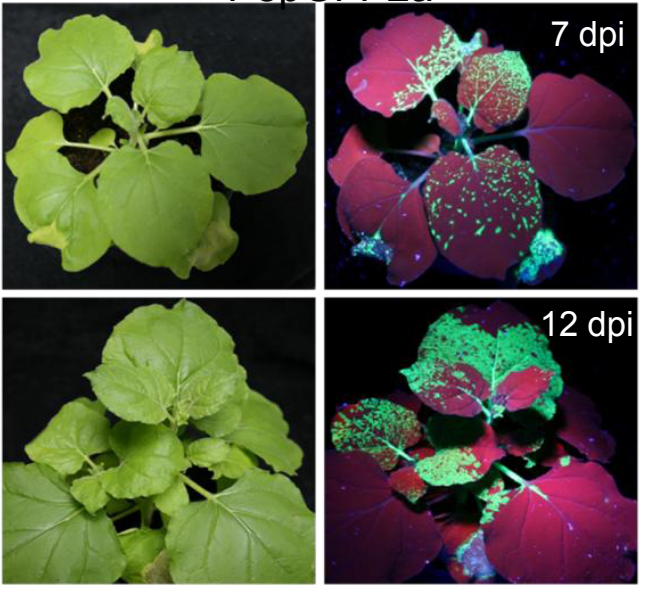

C

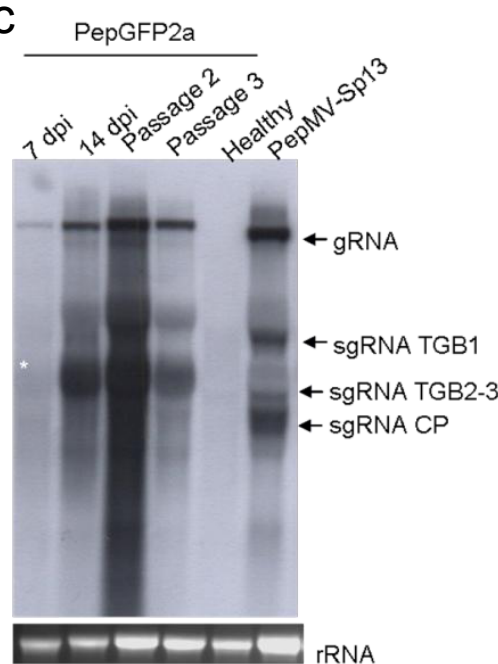

d

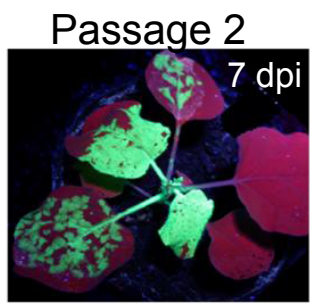

Passage 3

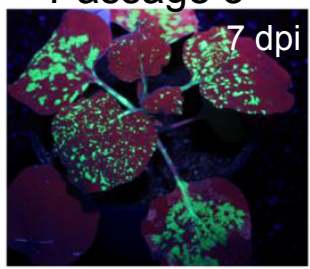

Figure 5 PepMV vector including a GFP:CP fusion separated by the FMDV 2A catalytic peptide sequence. (a) Schematic representation of PepGFP2a. The position of the 2A sequence insertion is indicated. (b) Fluorescence in systemically infected $\mathrm{N}$. benthamiana leaves from plants agroinfiltrated with pBPepGFP2a. (c) Northern blot of leaf tissue hybridized with a CP-specific RNA probe to determine PepGFP2a stability. Total RNA was prepared from agroinfiltrated and systemically-infected leaves showing fluorescence at 7 and 14 dpi, and from plants mechanically inoculated with PepGFP2a at $7 \mathrm{dpi}$ after the second and third passages. The band corresponding to the GFP-CP sgRNA is marked with star. Ethidium bromide-stained rRNA is shown (bottom panels). (d) GFP expression after second and third passage of PepGFP2a. Plants were photographed under white and UV light at 7 and 12 dpi. 
including aerial and subterranean parts. Mild or no visible symptoms of infection were observed during the course of the experiment. Northern blots of total RNAs extracted from fluorescent leaves 3-16 dpi revealed a signal corresponding in size to the CP2aGFP sgRNA (Figure 5c, lanes 1 and 2, marked with star).

Because PepGFP2a was the most promising candidate vector so far, we analyzed the stability of the GFP gene during serial passages, noting that GFP fluorescence was visible for several weeks in plants that were agroinfiltrated directly. We found that GFP fluorescence was maintained for up to four passages (Figure 5d), and Northern blots carried out 7 days after each passage confirmed the structural integrity of the virus (Figure 5c). During the third passage, at $12 \mathrm{dpi}$, GFP fluorescence began to decline in the upper leaves of the inoculated plants; Northern blots of total RNAs extracted from these leaves revealed a signal corresponding to the predicted size of the wild type CP sgRNA (data not shown), indicating that the vectors did eventually lose their integrity.

\section{Expression of GFP from PepGFP2a}

Protein extracts from systemically-infected leaves by 10 dpi were analyzed by western blot using a CP-specific antibody to determine whether the fusion protein was correctly processed. This revealed the presence of both a fusion protein (GFP2aCP) and a free CP, as well as a third signal representing a protein slightly larger than the free CP (Figure 6a); possibly, this third band corresponds to GFP plus the 12 immunorreactive aminoterminal amino acids of the CP fused to GFP due to the way the construct was designed.

The levels of GFP expression in plants agroinfiltrated with PepGFP2a were then investigated by SDS-PAGE and western blot. Total protein extracted from systemically-infected leaves was separated by SDS-PAGE, and a strong band migrating in the position expected for free GFP was observed in the Coomasie-stained gel (Figure $6 \mathrm{~b}$, lanes 2 and 3). This band was missing from the total protein extract prepared from leaves systemically infected with wild-type PepMV. The identity of this band was confirmed by western blot using an anti-GFP antibody, revealing that fluorescent leaves contained significant amounts of free GFP protein (Figure 6c). Relatively little of the GFP2aCP fusion protein was detected, indicating that cleavage by the $2 \mathrm{~A}$ peptide was nearly complete. Based on these data, plus the results of quantitative ELISA experiments (data not shown), we estimated that expression levels of $0.2-0.4 \mathrm{~g} / \mathrm{kg}$ leaf tissue could be achieved in systemically-infected leaves.

\section{A VIGS vector based on PepMV}

Finally, we tested if PepMV could be used to generate a VIGS vector. A 335 bp fragment of the tomato phytoene

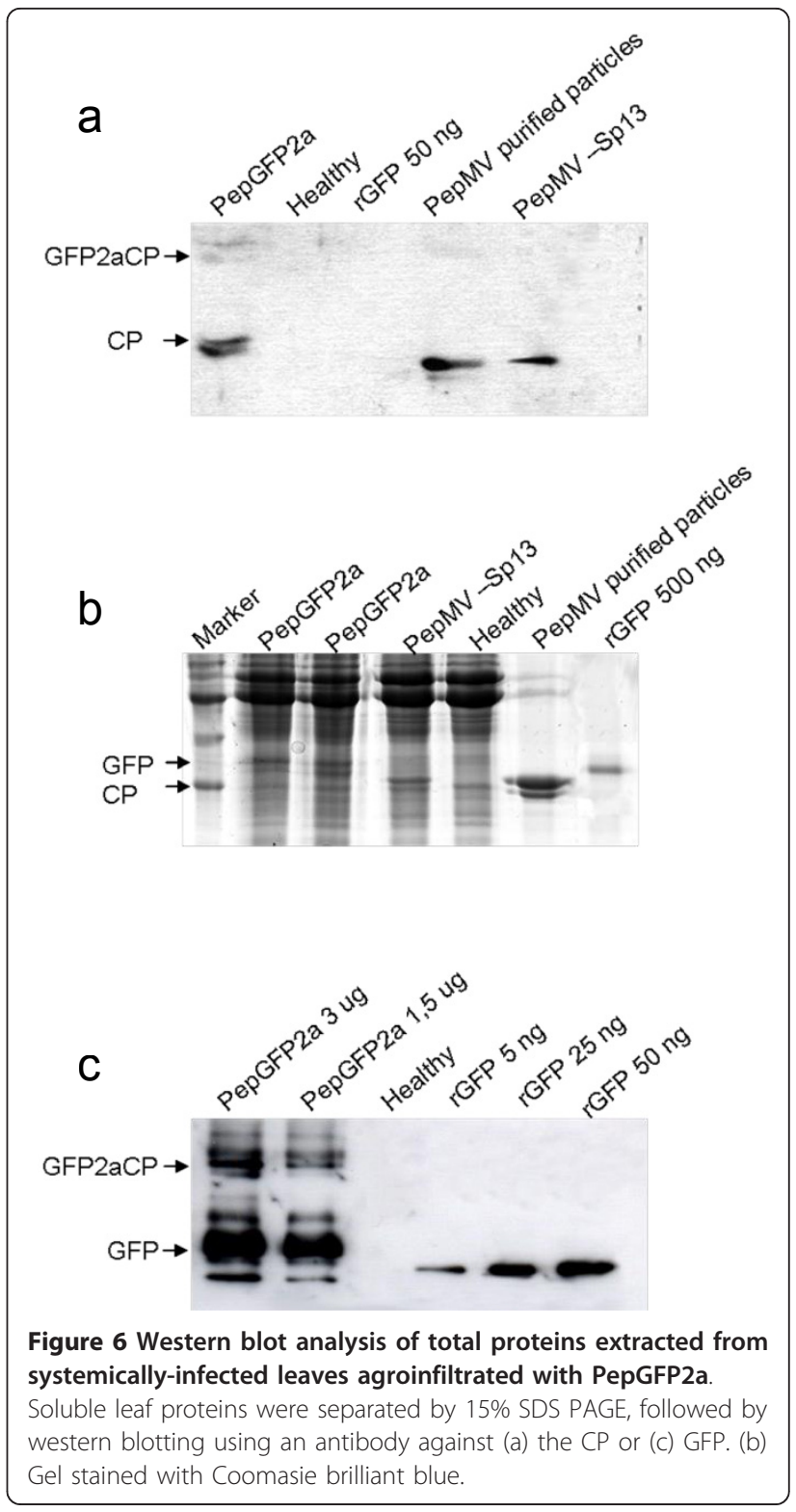

desaturase $(p d s)$ gene was inserted into the PepGFP2a vector, replacing the $g f p$ gene. The tomato $p d s$ fragment selected showed a sequence identity of $93 \%$ with the gene from $N$. benthamiana. Additionally, PepGFP2a was further modified to include additional cloning sites so that genes of interest could be inserted into AgeI and HpaI restriction sites. The resulting virus, PepPDS2a (Figure 7a), was used to infect $N$. benthamiana plants. All plants infiltrated with pBPepPDS2a began to display different photobleaching symptoms at 3-4 weeks postinfiltration (Figure 7b), attributed to the silencing of the endogenous $p d s$. The silencing phenotype was first detected in the upper uninoculated leaves. It appeared first as a yellow-white coloration along the veins and then, as silencing progressed, yellow-white areas became 


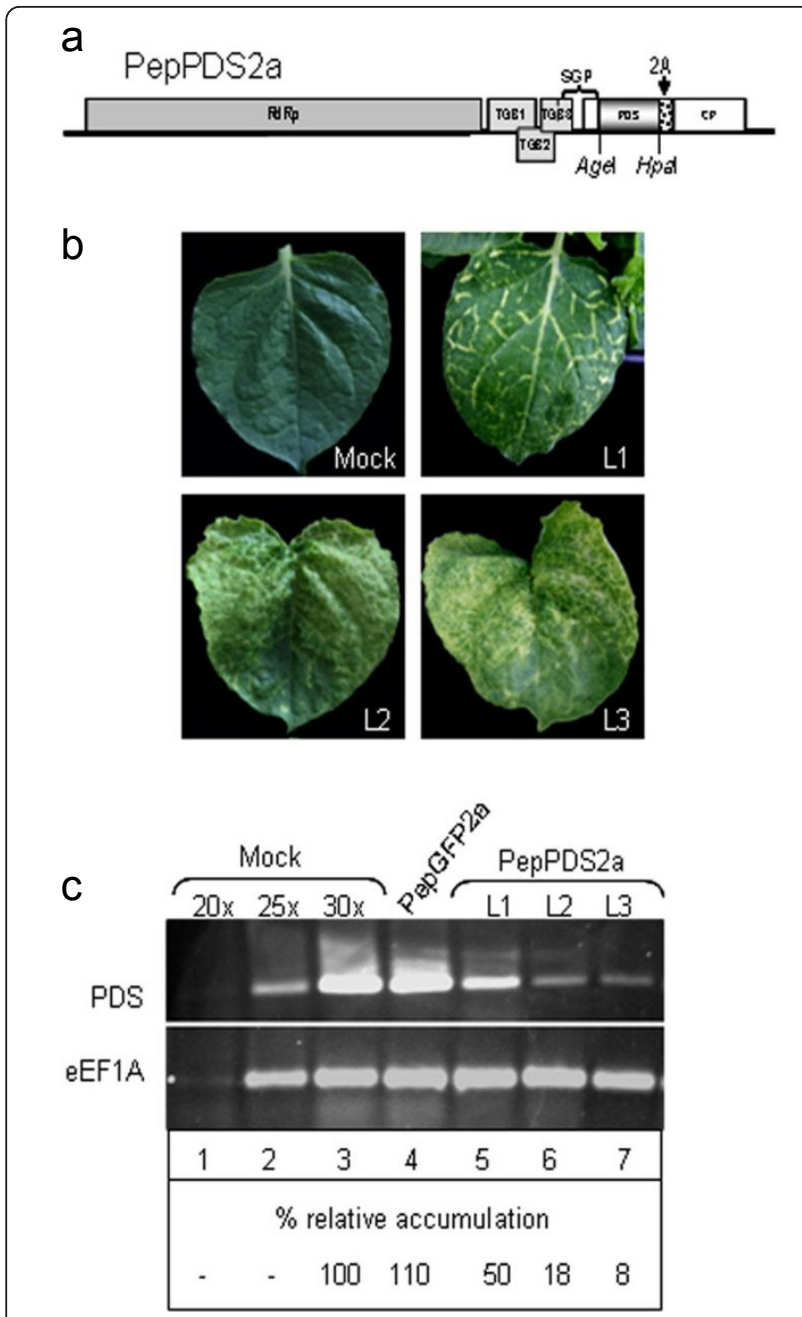

Figure 7 PepMV based gene silencing vector. (a) Schematic representation of PepPDS2a. (b) Representative leaves from $N$. benthamiana plants infected with the PepPDS2a vector showing different phenotypes of photobleaching (L1, L2 and L3). (c) Quantification of pds mRNA levels by semi-quantitative RT-PCR in plants inoculated with water (mock), pBPepGFP2a and pBPepPDS2a, as indicated. Lanes 1-3 correspond to mock-inoculated controls using 20, 25 and 30 amplification cycles. Thirty cycles were used for amplifications in lanes 4-7. L1, L2 and L3 correspond to the leaves with different phenotypes of photobleaching shown in the panel $b$. The relative (\%) accumulation of pds mRNA in relation to mockinoculated leaves is indicated below each lane.

visible in the leaves, covering with time almost the complete leaf.

To confirm VIGS at the molecular level, semi-quantitative RT-PCR was performed to compare the levels of $p d s$ transcripts present in leaves showing different photobleaching phenotypes (Figure 7c). The primers used for estimation of the $p d s$ mRNA levels were designed to anneal outside the region targeted for silencing, so that only the endogenous mRNA was probed. We used the eukaryotic elongation factor 1A (eEF1A)
mRNA as a constitutively expressed internal control. Quantification of the signal brightness of DNA bands revealed that the $p d s$ mRNA was on average $80-90 \%$ less abundant in photobleached leaves of plants infected with PepPDS2a than in mock-inoculated leaves or in leaves infected with PepGFP2a (Figure 7c, lanes 3 and 4, respectively). In contrast, similar levels of eEF1A mRNA were found in all cases (Figure 7c). As expected, $p d s$ mRNA levels were smaller in leaves exhibiting more complete photobleaching (L2 and L3 in Figure 7b) than in leaves where the silencing was restricted to the veins (L1 in Figure 7b) (Figure 7c). Taken together, these findings suggest that the photobleaching symptoms in the PepPDS2a-infiltrated $N$. benthamiana plants can be attributed to silencing of the plant $p d s$ mRNA. These results indicate that PepMV can be used as a viral vector to silence genes in $N$. benthamiana plants.

\section{Discussion}

In this work, two agroinfectious clones from two different PepMV genotypes (European and Chilean), able to initiate typical PepMV infections, were generated. We took advantage of these agroinfectious clones to develop a novel set of PepMV-based vectors. We investigated three key vector development strategies, namely replacement of the CP gene, duplication of the CP SGP and $\mathrm{CP}$ fusion using the FMDV 2A peptide. Although potexviruses are generally well-characterized, few studies have specifically focused on PepMV and little is known about the precise functions of its genes and proteins, and how these control its interactions with host plants. As part of our investigation, we have determined that the PepMV $\mathrm{CP}$ is required for cell-to-cell and long-distance movement, and we have gained insight into the minimal sequence required to act as a CP SGP.

We first sought to determine the relationship between $\mathrm{CP}$ expression and virus replication by constructing two PepMV mutants in which CP expression was abolished, in one case by a point mutation and in the other by the replacement of most of the CP ORF. Both mutants remained capable of replicating in protoplasts, confirming that the $\mathrm{CP}$ is not absolutely necessary for PepMV replication at the single-cell level. However, CP mutations in other potexviruses have been shown to reduce the amount of plus-sense RNA accumulating in protoplasts suggesting that, although the CP may not be essential for virus replication in single cells, it may stimulate this process $[49,50,55]$. Our results appear to rule out a direct and specific role of the $\mathrm{CP}$, so we speculate that the point mutation in PepXL6agg may increase the rate of RNA degradation because of its untranslatable 3' end, as shown for many other plant mRNAs [56]. However, while our protoplast transfections were indicative, they were not strictly quantitative, 
so without additional experiments we cannot completely exclude the possibility that the $\mathrm{CP}$ may influence the accumulation of PepMV RNA at the single-cell level.

Agroinfiltration of $N$. benthamiana leaves with pBPepGFP $\triangle \mathrm{CP}$ showed that although CP-deficient PepMV mutants were competent for replication, they were unable to achieve cell-to-cell movement. This phenomenon is well documented in other potexviruses $[49,50]$. The movement of PepMV-CP mutants could be restored by providing $\mathrm{CP}$ in trans but there were only few infection foci, suggesting that PepGFP $\triangle \mathrm{CP}$ was able to initiate infection foci but with a low efficiency. Movement-deficient TMV expression vectors delivered into $N$. benthamiana leaves by agroinfiltration also initiated replication rather inefficiently, a phenomenon that was attributed to improper processing of the primary transcript in the plant nucleus [57]. The authors showed that the addition of introns and the removal of putative splicing sites increased the proportion of infected cells from $0.6 \%$ to $96 \%$, and it is possible that the same strategy might improve the efficiency of PepMV-based vectors.

We constructed further PepMV vectors by duplicating the putative CP SGP and using this additional promoter to drive the GFP gene. Two vectors were prepared (pBPep501 and pBPep5.128) and both infected $N$. benthamiana leaves efficiently and were capable of systemic movement, resulting in strong GFP expression throughout the plant. However, this SGP duplication made the vector unstable and possibly prone to recombination; homologous recombination has been observed in many other viral vectors, resulting sooner or later in transgene elimination [33,38,58-61], although PVXbased vectors are remarkably stable [16]. Dragicci and Varrelamann [62] have shown that the frequency of transgene elimination in PVX vectors is proportional to the length of the homologous sequence available for template switching. Since our PepMV SGP is twice the size of the equivalent sequence in PVX vectors, that could be the reason for the observed instability [16]. The duplicated PepMV SGP contained all the cis-acting elements necessary for transgene expression. We attempted to increase the stability of the vector by removing potentially unnecessary downstream sequences from the duplicated SGP. Unfortunately, this size reduction reduced the level of virus RNA produced in infected plants, suggesting that the sequences between positions +12 and +36 are necessary for optimal SGP activity. Sequences within the CP ORF are also required for maximum sgRNA production in other viruses [63], usually by acting as enhancers $[64,65]$.

Stability has been improved in other vectors with duplicated SGPs by using heterologous promoters to reduce the likelihood of recombination $[59,63,65,66]$.
Accordingly, we generated a hybrid vector containing the SGPs from two distantly related PepMV isolates, PepMV-Sp13 and PepMV-Ps5. These sequences had an overall identity of $48 \%$, although they contained a highly conserved 22-nt region at the 5 ' end including the potexvirus-specific octanucleotide motif discussed above. Although such heterologous sequences have increased the stability of TMV vectors [63], they had little impact in the case of PepMV: the hybrid vector suffered transgene loss at 7-10 dpi, similarly as with the duplicated SGP vectors. In agreement with this result, Nagy and Bujarski [67] showed that as little as $15 \mathrm{nt}$ of sequence identity can promote homologous recombination in a brome mosaic virus vector. On the other hand, Dragicci and Varrelmann [62] provided evidence that RNA secondary structure was required to achieve precise homologous recombination. The PepMV CP SGP region potentially forms several stem-loops structures around the octanucleotide motif (data not shown) that may be involved in recombination events causing transgene elimination.

The FMDV 2A protease sequence has been used with success to overcome the problem of homologous recombination in viral vectors $[34,68]$ and has been shown to work in the context of both PVX and CPMV [34,69]. We therefore developed a further vector (PepGFP2a) in which GFP is expressed fusioned with the CP, with an intervening 2A peptide. This strategy appeared to solve our instability problems observed with the other vectors. Although PepGFP2a spread more slowly than Pep501, systemic infection was nevertheless observed by $7 \mathrm{dpi}$, and GFP fluorescence persisted for several weeks. PepGFP2a was stably propagated through up to four serial passages, each passage resulting in a similar systemic infection characterized by long-lasting uniform fluorescence, with instability events only starting to occur at $\sim 12 \mathrm{dpi}$ in the third passage. This instability might result from the presence of a 36-nt duplication within the CP gene. Western blot analysis of infected plants showed that the fusion protein was expressed and correctly processed, confirming that the $2 \mathrm{~A}$ protease was efficient in the context of PepMV infection (free CP was approximately tenfold more abundant than the GFP2aCP fusion protein showing that most of the fusion protein was fully processed). GFP accounted for 0.2-0.4 $\mathrm{g} / \mathrm{kg}$ of fresh tissue, comparable to the yields reported for CPMV [32] and early versions of TMV [70]. These yields are already sufficient for most applications, although they are not as high as those reported for highly-developed systems $[35,39,71,72]$. We expect that further refinement of the vector (e.g., altering the viral sequence to improve mRNA processing after agroinoculation, improving inoculation methods and other parameters) $[44,57]$ will lead to increased expression 
levels. The data presented here suggest that our system can be used for the expression of proteins that are at least as large as GFP. For the expression of larger proteins, systems based on "deconstructed" vectors (e.g., providing the CP in trans) could be explored [73].

The experiments carried out with PepGFP2a showed that PepMV viral vectors induce no or mild symptoms in the infected plants and that PepMV is distributed in most parts of the plants, infecting roots and flowers. These characteristics make PepMV an attractive system to be used as a tool for silencing genes in different tissues. Therefore, we constructed PepPDS2a and demonstrated that this vector is also suitable for VIGS experiments. Plants infected with PepPDS2a, carrying a fragment of the tomato $p d s$ gene, showed a clear bleaching phenotype visible at $21-28$ dpi. Molecular analysis showed successful $p d s$ silencing in stronger bleached yellow-white leaves, whereas in the leaves with bleaching occurring only in their veins silencing was less efficient, showing a clear correlation between phenotypes and mRNA levels. The fact that silencing was not equally efficient in different leaves is probably due to an unequal distribution of the virus in the infected tissues. It is noteworthy that the silencing capacity of our PepMV-based VIGS vector is possibly higher, because the $p d s$ fragment (from tomato) inserted into the vector had only $93 \%$ of nucleotide identity with the homologous gene from $N$. benthamiana. Moreover, it has been demonstrated that the length and the position of the insert with respect to the full-length cDNA can affect silencing efficiency of a particular vector [74]. Therefore these aspects need to be taken into account in the design of new and specific PepMV-based VIGS vectors.

\section{Conclusions}

In conclusion, we have demonstrated that PepMV can be used as an expression vector for recombinant protein expression in plants. Two distinct vector systems were envisaged, one based on an autonomous viral vector and another based on a "deconstructed" virus, where the CP is provided in trans $[39,40,75]$. Such vectors could serve for multiple purposes including the analysis of gene functions in planta, the expression of valuable recombinant proteins and the dissection of PepMV wild-type functions.

\section{Materials and methods}

\section{PepMV agroinfectious clones}

The full-length cDNAs of PepMV-Sp13 [13] and PepMV-Ps5 [3] were generated by RT-PCR using primers CE-42 (5'-CGC GGA TCC GGA AAA CAA AAT AAA TAA-3') or CE-292 (5'-GGG CCC GGA AAA CAA AAC ATA ACA C-3') and CE-43 (5'-(A) ${ }_{20}$ CCC
GGG CCC GCG GTA CCC C-3'), corresponding to the 5 ' and 3' termini of the $\mathrm{Sp} 13$ and Ch2 genomic RNAs, respectively, and including the necessary restriction sites (underlined). First strand cDNAs were synthesized using primer CE-43 and the SuperScript First-Strand Synthesis System for RT-PCR (Invitrogen), and amplified with Pyrobest polymerase (Takara). The resulting DNA fragments were purified and inserted into the pTOPO-XL vector (Invitrogen), generating constructs pTPepXL6 and pTPepPs5. The pTPepXL6 construct was inserted between the BamHI and XmaI sites of pBIN61 [47] to generate pBPepXL6, whereas pTPepPs5 was inserted at the BamHI site of the same vector to generate pBPepPs5. Constructs for in vitro transcription using the $\mathrm{T} 7$ promoter were produced by amplifying the viral insert in PepXL6 with forward primer CE-484 (5'-AAT ACG ACT CAC TAT AGG GAA AAC AAA $\overline{\text { ATA }}$ AAT AA-3'; T7 promoter sequence underlined) and reverse primer $\mathrm{CE}-43$.

\section{DNA constructs for PepMV mutants and vectors}

All PepMV mutants and vectors were constructed using standard overlapping PCR and molecular cloning methods [76]. For the construction of pT7PepXL6agg, two overlapping DNA fragments were amplified in separate PCRs. In PCR 1, a DNA fragment containing the replicase and the TGB genes and the first $11 \mathrm{nt}$ of the CP gene was amplified from pTPepXL6 using the primers CE-42 and CE-199 (5'-CAA TAA ACA ATC AgG CCT GAC AC-3'); the mutated nucleotide is shown in lower case within the context of the original ATG triplet, underlined). In PCR 2, a DNA fragment containing the CP gene and the 3' UTR was amplified with the primers CE-198 (reverse complement of CE-199) and CE-43. These overlapping fragments were mixed and amplified in a third PCR to produce a full-length DNA fragment using primers CE-484 and CE-43, complementary to the ends of the two initial fragments. The resulting PCR product was inserted into pTOPO-XL.

For the construction of pBPepGFP $\triangle C P$ and pT7PepGFP $\triangle C P$, the same steps as above were carried out amplifying two overlapping DNA fragments in separate PCRs. In PCR 1, a DNA fragment covering the 3' end of TGB1 though to the 5' end of the CP gene was amplified using pTPepXL6 as the template and the primers Pep303 (5'-CGG AAT TGC AGG CAC TGG G3') and CE-458 (5'-GTT GCT GCC ACT TCA AGT AGG AGT AAA GGA GAA GAA-3', including the first 18 nt of the GFP gene). CE-458 places the GFP ORF 36 nt downstream of the first CP codon. In PCR 2, a DNA fragment containing the GFP gene followed by the PepMV 3' UTR was amplified using primers CE-459 (reverse complement of CE-458) and CE-43. The overlapping products were mixed and amplified into a full- 
length DNA fragment using primers Pep303 and CE-43. The final PCR product was either inserted into pT7PepXL6agg using the XmnI and XmaI sites to produce pT7PepGFP $\triangle \mathrm{CP}$, or inserted into pTPepXL6 using the same restriction sites followed by subcloning into the BamHI and XmaI sites of pBPepXL6 to obtain pBPepGFP $\triangle$ CP.

For the construction of pBINCPep, a binary vector used to provide $\mathrm{CP}$ in trans, a DNA fragment corresponding to the PepMV CP gene was amplified using pTPepXL6 as the template and primers CE-356 (5'-CGC GGA TCC CAA TCA TGC CTG ACA C-3') and oligo $\mathrm{d}(\mathrm{T})$. The PCR product was introduced into the SmaI site of the binary vector pBIN61.

Overlapping PCR was also used to construct pBPep5.128. In PCR 1, a DNA fragment covering the 3' end of TGB1 through to the 3' end of the CP gene was amplified using pTPepXL6 as the template and primers Pep303 and CE-504 (5'-GAA AAC TTA ACC CGT TCC AAG TTA AAG TTC AGG GGG TG-3', including $18 \mathrm{nt}$ of the TGB4 (from 5563 to 5581), considered as the 5' end of the putative CP SGP). In PCR 2, a cDNA fragment containing the GFP gene fused to the PepMV3' UTR was amplified using primers CE-503 (reverse complement of CE-504) and CE-43. These PCR products were mixed and amplified in the third PCR with primers Pep303 and CE43. The resulting fragment was introduced into the XmnI and XmaI sites of pTPepXL6, and a BamHI/XmaI fragment from this construct was introduced into the BamHI and XmaI sites of the binary vector pBPepXL6. For the construction of pBPep501 by overlapping PCR, the same templates were used but in a different order. In this case, PepGFP $\triangle C P$ was used as a template in PCR 1 and PTPepXL6 in PCR 2, using the complementary primers CE-502 (5'- GAT GAA CTA TAC AAA TAA CTT GGA ACG GGT TAA GTT TTC-3') and CE-501 (5'- GAT GAA CTA TAC AAA TAA CTT GGA ACG GGT TAA GTT TTC-3') corresponding to the last $18 \mathrm{nt}$ of the GFP gene and the first 18 nt of TGB4 (from 5563 to 5581). The resulting fragment was transferred to pTPepXL6 and then to pBPepXL6 as described above.

For the construction of pBPep352 and pBPep640, the procedures and templates were the same as those used to produce pBPep501. Two consecutive sets of overlapping PCR were performed. The first was similar to that used to prepare pBPepGFP $\triangle \mathrm{CP}$ but with different complementary primers. For pBPep352, the primers were CE-352 (5'-CCT GAC ACA ACA CCT GTT CCG CGG ATG AGT AAA GGA-3') and CE-350 (reverse complement of CE-352). For pBPep640, the primers were CE640 (5'-AAC AAT CAT GCC TGA CAC AAT GAG TAA AGG AGA AGA-3') and CE-641 (reverse complement of CE-641). The second overlapping PCR and subsequent cloning steps followed were exactly as described for pBPep5.128.

Two consecutive sets of overlapping PCRs were also required for the construction of pBPep505. The first of overlapping PCRs was used to generate the $\mathrm{SGP}_{\mathrm{Ps} 5}$ - $^{-}$ GFP-3' UTR fragment. In PCR 1, a DNA fragment containing the putative $\mathrm{SGP}_{\mathrm{Ps} 5}$ was amplified using pTPepPs5 as the template and primers CE-497 (5'-GCA ATA AAC TTC TCC CCT TGG AAC GGG TTA AGT-3', corresponding to $18 \mathrm{nt}$ of PepMV-Sp13 TGB4 (from 5545 to 5563) and $18 \mathrm{nt}$ of PepMV-Ps5 TGB4 (from 5553 to 5571)) and CE-498 (5'-GCT TCT AAC CCA TCA GAT ATG AGT AAA GGA GAA GAA-3', corresponding to $18 \mathrm{nt}$ of the CP gene (from 5651 to 5669) and 18 nt of the GFP gene). In PCR 2, a DNA fragment comprising GFP fused to the PepMV-3' UTR was amplified using primers CE-499 (the reverse complement of CE-498) and CE-43. The overlapping products were joined in a third PCR using primers CE-497 and CE-43. The second set of overlapping PCRs was as described for the construction of pBPep5.128 although

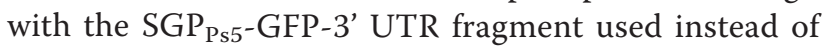
$\mathrm{pBPepGFP} \triangle \mathrm{CP}$ in PCR 2. The subsequent cloning steps were the same as those used in the construction of pBPep5.128.

Two consecutive sets of overlapping PCRs were also used for the construction of PepGFP2a (pBPepGFP2a). In PCR 1, a DNA fragment covering the 3' end of TGB1 through to the 5 ' end of the $\mathrm{CP}$ gene was amplified using pTPepXL6 as the template and primers Pep303 and CE-458. In PCR 2, a DNA fragment containing the GFP gene and 2A sequence was amplified using CE-459 (the reverse complement of CE-458) and CE-665 (5'GAG TCC AAC CCT GGG CCT GAC ACA ACA CCT GTT-3', corresponding to last 18 nucleotides of $2 \mathrm{~A}$ sequence and nt 4-21 of the $\mathrm{CP}$ ). Because $2 \mathrm{~A}$-mediated processing requires a proline to follow the $2 \mathrm{~A}$ sequence (NFDLLKLAGDVESNPG), it was inserted between the last codon of the GFP gene and the second codon of the CP gene, which specifies a proline residue. The products were mixed and amplified to obtain the full-length DNA using primers Pep303 and CE-665. The second set of overlapping PCRs was similar to those used in the construction of Pep501, although the fragment obtained with primers Pep303 and CE-665 was used as the template in PCR 1. and in PCR 2, a DNA fragment containing the CP gene and 3' UTR was amplified using primers CE-664 (the reverse complement of CE-665) and $\mathrm{CE}-43$. The subsequent cloning steps were as described for the construction of pBPep5.128.

The design of pBPepPDS2a was basically the same as for pBPepGFP2a but in this case we introduced a portion of the tomato $p d s$ sequence [from 962 to 1319; GeneBanK 544073] flanked by sites AgeI and HpaI 
instead of the $g f p$ sequence (Figure 7a). For the construction of pBPepPDS2a, a DNA fragment reaching from the 3' end of TGB1 until the 3' UTR with a XmaI site inmediatly after the poly(A) tail, and including the tomato $p d s$ sequence in-between as mentioned before, was synthesized by GeneScript (New Jersey, USA). This fragment was digested with XmnI and XmaI and introduced into the same sites of pTPepXL6, and further subcloned into the binary vector pBIN61 using the BamHI/XmaI restriction sites, resulting in pBPepPDS2a.

\section{In vitro transcription and protoplast transfection}

Plasmids pT7PepXL6, pT7PepXL6agg and pT7PepGFP $\Delta C P$ were linearized with $K p n I$ and transcribed in vitro in the presence of cap analog $\left(\mathrm{m}^{7} \mathrm{G}^{5} \mathrm{pppNp}\right)$ (Promega) using RiboMAX ${ }^{\mathrm{TM}}$ Large Scale RNA Production Systems (Promega) according to the manufacturer's instructions. $N$. benthamiana plants (4-5 weeks old), grown in a growth chamber (16-h photoperiod, $22^{\circ} \mathrm{C}$ ), were used for preparation of mesophyll protoplasts as described by Weston and Turner [77]. Approximately $10^{6}$ protoplasts were electroporated with $10 \mu \mathrm{g}$ of in vitro transcribed RNA. The protoplasts were incubated in the dark at $25^{\circ} \mathrm{C}$ and harvested after $24 \mathrm{~h}$ for RNA extraction.

\section{Inoculation and fluorescence visualization}

Agrobacterium tumefaciens strain C58C1 was transformed with the different PepMV constructs cloned in binary vector pBIN61 by electroporation. Overnight cultures $(3 \mathrm{ml})$ were centrifuged at 2,500 $\mathrm{g}$ for $10 \mathrm{~min}$. The pellet was resuspended in the same volume of $10 \mathrm{mM}$ MES (pH 5.5), $10 \mathrm{mM} \mathrm{MgSO}_{4}$ and $100 \mu \mathrm{M}$ acetosyringone. Leaves of $N$. benthamiana plants from the greenhouse were infiltrated using a syringe without a needle. For trans-complementation analysis, A. tumefaciens cultures were mixed in a 1:1 ratio. If P19 was used in the experiments, A. tumefaciens cultures containing the pBP19 vector were mixed with those containing the PepMV constructs at a 1:3 ratio. Mechanical inoculation was carried out by homogenizing leaf tissue in $30 \mathrm{mM}$ sodium phosphate, $\mathrm{pH}$ 8.0, followed by rubbing 3-weekold carborundum-dusted $N$. benthamiana leaves with the extract. $N$. benthamiana plants (4-5 weeks old) were grown in a growth chamber $\left(16-\mathrm{h}\right.$ photoperiod, $25^{\circ} \mathrm{C}$ for GFP expression or $22^{\circ} \mathrm{C}$ to induce silencing). Leaves were viewed under UV light $(365 \mathrm{~nm})$ provided by a handheld lamp (Blak Ray B100-AP lamp, UV products, Upland, CA 91786, USA). For confocal imaging, $0.5 \mathrm{~cm}^{2}$ squares of leaf tissue were prepared, mounted in water and imaged using a Leica TCS SP2 (Leica Microsystems, Germany) inverted confocal laser scanning microscope. The fluorescence intensity was measure using Qwin V3 (Leica) image analysis software.

\section{RNA and protein analysis}

RNA was extracted from protoplasts or leaf tissue using TRI-Reagent (Sigma Chemical Co., St. Louis, MO) and separated in agarose gels containing formaldehyde. The RNA was transferred to positively charged nylon membranes (Roche, Indianapolis, IN, USA), which were hybridized with digoxigenin-labeled probes (prepared by in vitro transcription) specific for PepMV replicase, PepMV $\mathrm{CP}$ or GFP [76]. Chemiluminescent detection was carried out using the reagents and protocols supplied in the DIG-labeling and detection kit (Roche Diagnostics). For protein extractions, $100 \mathrm{mg}$ samples of fluorescent $N$. benthamiana leaves were ground in $200 \mu$ l protein extraction buffer (0.1 M Tris, $\mathrm{pH}$ 8.0, $0.125 \mathrm{mM}$ 2-mercaptoethanol, 200 mM PMSF, 10\% glycerol). Total soluble protein content was determined using the Bradford assay. Crude extracts were mixed with $4 \times$ loading buffer and separated by SDS-PAGE followed by staining with Coomassie Brilliant Blue or by electrotransfer to nitrocellulose membranes. Blots were probed with monoclonal antibodies raised in rabbits against PepMV CP (AC Diagnostics, Fayetteville, USA) or in mouse against GFP (Clontech, Palo Alto, CA, USA) followed by detection with anti-rabbit or anti-mouse immunoglobulin G (IgG) coupled to horseradish peroxidase (Promega) and chemiluminescence (ECL Western Blotting and Protein Analysis System, Amersham Biosciences). The image was taken with the GeneSnap software on a G:BOX (Syngene, Cambrige, UK) and signal intensity of DNA bands quantified using the Gene Tools software (Syngene, Cambrige, UK).

\section{Semiquantitative RT-PCR analysis}

For semiquantitative RT-PCR analysis, leaves expressing different bleaching phenotypes were harvested at 5 and 6 weeks post-infiltration. Total RNA was extracted using Tri-Reagent and samples were treated with RNase-free Dnase I (Roche) prior to reverse-transcription with the One-Step RT-PCR kit (Qiagen, Valencia, CA, USA) according to the supplier's instructions. Primers CE-787 (5'-TTT CCA AAA GTT GGA GAA GC3') and CE-788 (5'-TCA TGT TGT CAA AAC CCC AA-3') used to amplify the $p d s$ mRNA, annealed outside the region targeted for silencing. Semiquantitative RTPCR was performed as described by Wang et al. [78] using primers CE-780F (5'-ACA AAC CCC TCC GTC TTC CAC-3') and CE-781 (5'-CCC TAC TGG TTT GAC AAC TGA-3') to amplify eEF1A mRNA as internal, constitutively expressed control. RT-PCR reactions, in a final volume of $25 \mu \mathrm{l}$, contained $250 \mathrm{ng}$ of RNA and $0.6 \mu \mathrm{M}$ of each pair of primers. After 25 and 30 cycles, the PCR-generated fragments were analyzed in $1.5 \%$ agarose gels, and the intensity of the amplified fragments was quantified using GeneSnap sofware tools. 


\section{Acknowledgements}

M.C. Montesinos and J. Tudela provided excellent technical assistance. R. Twyman checked the English. Financial support was provided by grants PET2008_0239 (Ministerio de Ciencia e Innovación, Spain) and 14/08 (Consejería de Universidades, Empresa e Investigación, Región de Murcia, Spain). R.N.S. was the recipient of a PhD fellowship from Fundación Seneca (Murcia, Spain). P.G. was supported by a "Juan de la Cierva" post-doctoral contract from Ministerio de Ciencia e Innovación (MICINN, Spain). We thank David Baulcombe for providing a DNA clone for suppressor of gene silencing P19.

\section{Author details}

'Departamento de Biología del Estrés y Patología Vegetal, Centro de Edafología y Biología Aplicada del Segura (CEBAS)- CSIC, PO Box 164, 30100 Espinardo, Murcia, Spain. ${ }^{2}$ Bioprodin SL, Edificio CEEIM, Campus de Espinardo s/n, 30100 Espinardo, Murcia, Spain. ${ }^{3}$ Department of Zoology, Oxford University, Oxford OX1 3PS, UK.

\section{Authors' contributions}

RNS designed the experiments, carried out most of the experimental work, wrote the manuscript and co-participated in the conception of the work. PG developed the agroinfectious clone for PepMV-Ps5. VT co-participated in the conception of the work and supervised the laboratory work for the generation of the DNA constructs. MAA is the principal investigator, conceived the project and supervised the experiments and the writing of the manuscript. All authors read and approved the final manuscript.

\section{Competing interests}

The authors declare that they have no competing interests.

Received: 7 February 2011 Accepted: 11 March 2011

Published: 11 March 2011

\section{References}

1. Cotillon AC, Girard M, Ducouret S: Complete nucleotide sequence of the genomic RNA of a French isolate of Pepino mosaic virus (PepMV). Arch Virol 2002, 147:2231-2238.

2. French CJ, Bouthillier M, Bernardy M, Ferguson G, Sabourin M, Johnson RC, Masters C, Godkin S, Mumford R: First Report of Pepino mosaic virus in Canada and the United States. Plant Dis 2001, 85:1121-1121.

3. Gomez P, Sempere RN, Elena SF, Aranda MA: Mixed Infections of Pepino Mosaic Virus Strains Modulate the Evolutionary Dynamics of this Emergent Virus. J Virol 2009, 83:12378-12387.

4. Hanssen IM, Thomma BPHJ: Pepino mosaic virus: a successful pathogen that rapidly evolved from emerging to endemic in tomato crops. Mol Plant Pathol 2010, 11:179-189.

5. Hasiów-Jaroszewska B, Pospieszny H, Borodynko N: New necrotic isolates of pepino mosaic virus representing the Ch2 genotype. J Phytopathol 2009, 157:494-496

6. Ling KS, Carpenter L: Pepino mosaic virus, an emerging disease in greenhouse tomato production worldwide: is seed responsible? Acta Horticulturae 2005, 695:43-50.

7. Ling K-S, Wintermantel WM, Bledsoe M: Genetic Composition of Pepino mosaic virus Population in North American Greenhouse Tomatoes. Plant Dis 2008, 92:1683-1688.

8. Maroon-Lango CJ, Guaragna MA, Jordan RL, Hammond J, Bandla M, Marquardt SK: Two unique US isolates of Pepino mosaic virus from a limited source of pooled tomato tissue are distinct from a third (European-like) US isolate. Arch Virol 2005, 150:1187-1201.

9. Mumford RA, Metcalfe EJ: The partial sequencing of the genomic RNA of a UK isolate of Pepino mosaic virus and the comparison of the coat protein sequence with other isolates from Europe and Peru. Arch Virol 2001, 146:2455-2460

10. Pagan I, Cordoba-Selles MD, Martinez-Priego L, Fraile A, Malpica JM, Jorda C, Garcia-Arenal F: Genetic structure of the population of Pepino mosaic virus infecting tomato crops in Spain. Phytopathology 2006, 96:274-279.

11. van der Vlugt RAA, Stijger CCMM, Verhoeven JTJ, Lesemann DE: First Report of Pepino Mosaic Virus on Tomato. Plant Dis 2000, 84:103-103.
12. Jones RAC, Koening R, Leseman D-E: Pepino mosaic virus, a new potexvirus from pepino (Solanum muricatum). Ann Appl Biol 1980, 94:61-68.

13. Aguilar JM, Hernandez-Gallarod MD, Cenis JL, Lacasa A, Aranda MA: Complete sequence of the Pepino mosaic virus RNA genome. Arch Virol 2002, 147:2009-2015.

14. Verchot-Lubicz J, Ye CM, Bamunusinghe D: Molecular biology of potexviruses: recent advances. J Gen Virol 2007, 88:1643-1655.

15. Verchot J, Angell SM, Baulcombe DC: In Vivo Translation of the Triple Gene Block of Potato Virus $X$ Requires Two Subgenomic mRNAs. J Virol 1998, 72:8316-8320.

16. Baulcombe DC, Chapman S, Cruz S: Jellyfish green fluorescent protein as a reporter for virus infections. Plant J/ 1995, 7:1045-1053.

17. Yang CD, Liao JT, Lai CY, Jong MH, Liang CM, Lin YL, Lin NS, Hsu YH, Liang SM: Induction of protective immunity in swine by recombinant bamboo mosaic virus expressing foot-and-mouth disease virus epitopes. Bmc Biotechnology 2007, 7:62.

18. Canizares MC, Lomonossoff GP, Nicholson L: Development of cowpea mosaic virus-based vectors for the production of vaccines in plants. Expert Rev Vaccines 2005, 4:687-697.

19. Canizares MC, Nicholson L, Lomonossoff GP: Use of viral vectors for vaccine production in plants. Immunol Cell Biol 2005, 83:263-270.

20. Daniell $H$, Singh ND, Mason $H$, Streatfield SJ: Plant-made vaccine antigens and biopharmaceuticals. Trends Plant Sci 2009, 14:669-679.

21. Lico C, Chen Q, Santi L: Viral vectors for production of recombinant proteins in plants. J Cell Physioly 2008, 216:366-377.

22. Zhang Y, Li J, Pu H, Jin J, Zhang X, Chen M, Wang B, Han C, Yu J, Li D: Development of Tobacco necrosis virus $A$ as a vector for efficient and stable expression of FMDV VP1 peptides. Plant Biotechnol J 2010, 8:506-523.

23. Fu D, Zhu B, Zhu H, Jiang W, Luo Y: Virus-induced gene silencing in tomato fruit. Plant J 2005, 43:299-308.

24. Zhang CQ, Bradshaw JD, Whitham SA, Hill JH: The Development of an Efficient Multipurpose Bean Pod Mottle Virus Viral Vector Set for Foreign Gene Expression and RNA Silencing. Plant Physiol 2010, 153:52-65.

25. Kumagai MH, Donson J, della-Cioppa G, Harvey D, Hanley K, Grill LK Cytoplasmic inhibition of carotenoid biosynthesis with virus-derived RNA. Proc Natl Acad Sci USA 1995, 92:1679-1683.

26. Brigneti G, Martin-Hernandez AM, Jin HL, Chen J, Baulcombe DC, Baker B, Jones JDG: Virus-induced gene silencing in Solanum species. Plant J 2004, 39:264-272.

27. Liu Y, Schiff M, Dinesh-Kumar SP: Virus-induced gene silencing in tomato. Plant J 2002, 31:777-786.

28. Burch-Smith TM, Anderson JC, Martin GB, Dinesh-Kumar SP: Applications and advantages of virus-induced gene silencing for gene function studies in plants. Plant J 2004, 39:734-746.

29. Nagamatsu A, Masuta C, Senda M, Matsuura H, Kasai A, Hong J-S, Kitamura K, Abe J, Kanazawa A: Functional analysis of soybean genes involved in flavonoid biosynthesis by virus-induced gene silencing. Plant Biotechnol J 2007, 5:778-790.

30. Chapman S, Faulkner C, Kaiserli E, Garcia-Mata C, Savenkov El, Roberts AG, Oparka KJ, Christie JM: The photoreversible fluorescent protein iLOV outperforms GFP as a reporter of plant virus infection. Proc Natl Acad Sci USA 2008, 105:20038-20043.

31. Whitham SA, Yamamoto ML, Carrington JC: Selectable viruses and altered susceptibility mutants in Arabidopsis thaliana. Proc Natl Acad Sci USA 1999, 96:772-777.

32. Cañizares MC, Li L, Yolande P, Estratios T, George PL: A bipartite system for the constitutive and inducible expression of high levels of foreign proteins in plants. Plant Biotechnol J 2006, 4:183-193.

33. Dawson WO, Lewandowski DJ, Hilf ME, Bubrick P, Raffo AJ, Shaw JJ, Grantham GL, Desjardins PR: A tobacco mosaic virus-hybrid expresses and loses an added gene. Virology 1989, 172:285-292.

34. Gopinath K, Wellink J, Porta C, Taylor KM, Lomonossoff GP, van Kammen A: Engineering Cowpea Mosaic Virus RNA-2 into a Vector to Express Heterologous Proteins in Plants. Virology 2000, 267:159-173.

35. Marillonnet S, Giritch A, Gils M, Kandzia R, Klimyuk V, Gleba Y: In planta engineering of viral RNA replicons: Efficient assembly by recombination of DNA modules delivered by Agrobacterium. Proc Natl Acad Sci USA 2004, 101:6852-6857 
36. Zhang C, Ghabrial SA: Development of bean pod mottle virus-based vectors for stable protein expression and sequence-specific virusinduced gene silencing in soybean. Virology 2006, 344:401-411.

37. Chapman S, Kavanagh T, Baulcombe D: Potato virus $\mathrm{X}$ as a vector for gene expression in plants. Plant J 1992, 2:549-557.

38. Donson J, Kearney CM, Hilf ME, Dawson WO: Systemic expression of a bacterial gene by a tobacco mosaic virus-based vector. Proc Natl Acad Sci USA 1991, 88:7204-7208.

39. Fujiki M, Kaczmarczyk JF, Yusibov V, Rabindran S: Development of a new cucumber mosaic virus-based plant expression vector with truncated $3 a$ movement protein. Virology 2008, 381:136-142.

40. Gleba Y, Klimyuk V, Marillonnet S: Viral vectors for the expression of proteins in plants. Curr Opin Biotechnol 2007, 18:134-141.

41. Pogue GP, Lindbo JA, Garger SJ, Fitzmaurice WP: Making an ally from an enemy: Plant virology and the new agriculture. Annu Rev Phytopathol 2002, 40:45-74.

42. Sanchez-Navarro J, Miglino R, Ragozzino A, Bol JF: Engineering of Alfalfa mosaic virus RNA 3 into an expression vector. Arch Virol 2001, 146:923-939.

43. Scholthof KB, Mirkov TE, Scholthof HB: Plant virus gene vectors: Biotechnology applications in agriculture and medicine. Genet eng 2002, 24:67-85.

44. Gleba Y, Klimyuk V, Marillonnet S: Magnifection-a new platform for expressing recombinant vaccines in plants. Vaccine 2005, 23:2042-2048.

45. Musiychuk K, Stephenson N, Bi H, Farrance CE, Orozovic G, Brodelius M, Brodelius P, Horsey A, Ugulava N, Shamloul AM, Mett V, Rabindran S, Streatfield SJ, Yusibov V: A launch vector for the production of vaccine antigens in plants. Influ Other Respir Viruses 2007, 1:19-25.

46. Turpen TH, Turpen AM, Weinzettl N, Kumagai MH, Dawson WO: Transfection of whole plants from wounds inoculated with Agrobacterium tumefaciens containing cDNA of tobacco mosaic virus. $J$ Virol Methods 1993, 42:227-239.

47. Bendahmane A, Querci M, Kanyuka K, Baulcombe DC: Agrobacterium transient expression system as a tool for the isolation of disease resistance genes: application to the $R \times 2$ locus in potato. Plant J 2000, 21:73-81.

48. Voinnet $O$, Rivas $S$, Mestre $P$, Baulcombe $D$ : An enhanced transient expression system in plants based on suppression of gene silencing by the 19 protein of tomato bushy stunt virus. Plant J 2003, 33:949-956.

49. Chapman S, Hills G, Watts J, Baulcombe D: Mutational analysis of the coat protein gene of potato virus $\mathrm{X}$ : Effects on virion morphology and viral pathogenicity. Virology 1992, 191:223-230.

50. Lough TJ, Netzler NE, Emerson SJ, Sutherland P, Carr F, Beck DL, Lucas WJ, Forster RLS: Cell-to-cell movement of Potexviruses: Evidence for a ribonucleoprotein complex involving the coat protein and first triple gene block protein. Mol Plant-Microbe Interact 2000, 13:962-974.

51. Ozeki J, Hashimoto M, Komatsu K, Maejima K, Himeno M, Senshu H, Kawanishi T, Kagiwada S, Yamaji Y, Namba S: The N-terminal Region of the Plantago asiatica mosaic virus Coat Protein Is Required for Cell-to-Cell Movement but Is Dispensable for Virion Assembly. Mol Plant-Microbe Interact 2009, 22:677-685.

52. Hu B, Pillai-Nair N, Hemenway C: Long-distance RNA-RNA interactions between terminal elements and the same subset of internal elements on the potato virus $X$ genome mediate minus- and plus-strand RNA synthesis. RNA 2007, 13:267-280.

53. Skryabin KG, Morozov S, Kraev AS, Rozanov MN, Chernov B, K L, L I, Atabekov JG: Conserved and variable elements in RNA genomes of Potexviruses. FeBS Lett 1988, Lett 240:33-40.

54. Ryan MD, King AMQ, Thomas GP: Cleavage of foot-and-mouth disease virus polyprotein is mediated by residues located within a 19 amino acid sequence. J Gen Virol 1991, 72:2727-2732.

55. Forster RLS, Beck DL, Guilford PJ, Voot DM, Van Dolleweerd CJ, Andersen MT: The coat protein of white clover mosaic potexvirus has a role in facilitating cell-to-cell transport in plants. Virology 1992, 191:480-484

56. Shyu $A B$, Wilkinson MF, van Hoof $A$ : Messenger RNA regulation: to translate or to degrade. Embo J 2008, 27:471-481.
57. Marillonnet S, Thoeringer C, Kandzia R, Klymyuk V, Gleba Y: Systemic Agrobacterium tumefaciens-mediated transfection of viral replicons for efficient transient expression in plants. Nature biotechnol 2005, 23:718-723.

58. Dolja W, Herndon KL, Pirone TP, Carrington JC: Spontaneous mutagenesis of a plant potyvirus genome after insertion of a foreign gene. J Virol 1993, 67:5968-5975.

59. Haviv S, Galiakparov N, Goszczynski DE, Batuman O, Czosnek H, Mawassi M: Engineering the genome of Grapevine virus $A$ into a vector for expression of proteins in herbaceous plants. J Virol Methods 2006, 132:227-231.

60. Rabindran S, Dawson WO: Assessment of Recombinants That Arise from the Use of a TMV-Based Transient Expression Vector. Virology 2001, 284:182-189.

61. Zhao Y, Hammond J, Tousignant ME, Hammond RW: Development and evaluation of a complementation-dependent gene delivery system based on cucumber mosaic virus. Arch Virol 2000, 145:2285-2295.

62. Draghici H-K, Varrelmann M: Evidence for similarity-assisted recombination and predicted stem-loop structure determinant in potato virus X RNA recombination. J Gen Virol 2010, 91:552-562.

63. Shivprasad S, Pogue GP, Lewandowski DJ, Hidalgo J, Donson J, Grill LK, Dawson WO: Heterologous sequences greatly affect foreign gene expression in tobacco mosaic virus-based vectors. Virology 1999, 255:312-323.

64. Grdzelishvili VZ, Chapman SN, Dawson WO, Lewandowski DJ: Mapping of the Tobacco mosaic virus movement protein and coat protein subgenomic RNA promoters in vivo. Virology 2000, 275:177-192.

65. Lee YS, Hsu YH, Lin NS: Generation of subgenomic RNA directed by a satellite RNA associated with Bamboo mosaic potexvirus: Analyses of potexvirus subgenomic RNA promoter. J Virol 2000, 74:10341-10348.

66. Koenig R, Lesemann DE, Loss S, Engelmann J, Commandeur U, Deml G, Schiemann J, Aust H, Burgermeister W: Zygocactus virus X-based expression vectors and formation of rod-shaped virus-like particles in plants by the expressed coat proteins of Beet necrotic yellow vein virus and Soil-borne cereal mosaic virus. J Gen Virol 2006, 87:439-443.

67. Nagy PD, Bujarski JJ: Efficient system of homologous RNA recombination in brome mosaic virus: sequence and structure requirements and accuracy of crossovers. J Virol 1995, 69:131-140.

68. Mattion NM, Harnish EC, Crowley JC, Reilly PA: Foot-and-mouth disease virus $2 \mathrm{~A}$ protease mediates cleavage in attenuated Sabin 3 poliovirus vectors engineered for delivery of foreign antigens. J Virol 1996, 70:8124-8127.

69. Cruz SS, Chapman S, Roberts AG, Roberts IM, Prior DAM, Oparka KJ: Assembly and movement of a plant virus carrying a green fluorescent protein overcoat. Proc Natl Acad Sci USA 1996, 93:6286-6290.

70. Hamamoto $H$, Sugiyama $Y$, Nakagawa N, Hashida E, Matsunaga $Y$, Takemoto S, Watanabe Y, Okada Y: A new tobacco mosaic virus vector and its use for the systemic production of Angiotensin-l-converting enzyme inhibitor in transgenic tobacco and tomato. Biotechnol and Bioeng 1993, 11:930-932.

71. Giritch A, Marillonnet S, Engler C, van Eldik G, Botterman J, Klimyuk V, Gleba $Y$ : Rapid high-yield expression of full-size IgG antibodies in plants coinfected with noncompeting viral vectors. Proc Natl Acad Sci USA 2006, 103:14701-14706.

72. Green BJ, Fujiki M, Mett V, Kaczmarczyk J, Shamloul M, Musiychuk K, Underkoffler S, Yusibov V, Mett V: Transient protein expression in three Pisum sativum (green pea) varieties. Biotechnoly J/ 2009, 4:230-237.

73. Gleba $Y$, Marillonnet $S$, Klimyuk V: Engineering viral expression vectors for plants: the 'full virus' and the 'deconstructed virus' strategies. Curr Opin Biotechnol 2004, 7:182-188.

74. Liu E, Page J: Optimized cDNA libraries for virus-induced gene silencing (VIGS) using tobacco rattle virus. Plant Methods 2008, 4:5.

75. Sainsbury F, Lavoie PO, D'Aoustm MA, Vézina LP, Lomonossoff GP: Expression of multiple proteins using full-length and deleted versions of cowpea mosaic virus RNA-2. Plant Biotechnol J 2008, 6:82-92.

76. Sambrook J, Russell DW: Molecular cloning: A laboratory manual. Cold Spring Harbor, Cold Spring Harbor Press; 2001.

77. Weston JH, Turner RL: Analysis of Coat Protein Expression Cassettes in Protoplasts. In Plant Virology protocols: From Virus Isolation to Transgenic 
Resistance Methods in Molecular Biology. Volume 81. Edited by: Foster GD, Taylor SC. Totowa, NJ: Humana Press; 1998:301-305.

78. Wang T, lyer LM, Pancholy R, Shi X, Hall TC: Assessment of penetrance and expressivity of RNAi-mediated silencing of the Arabidopsis phytoene desaturase gene. New Phytol 2005, 167:751-760.

doi:10.1186/1746-4811-7-6

Cite this article as: Sempere et al:: Development of expression vectors based on pepino mosaic virus. Plant Methods 2011 7:6.

Submit your next manuscript to BioMed Central and take full advantage of:

- Convenient online submission

- Thorough peer review

- No space constraints or color figure charges

- Immediate publication on acceptance

- Inclusion in PubMed, CAS, Scopus and Google Scholar

- Research which is freely available for redistribution 